\title{
Homozygous 1B and 1BL/1RS Chromosome Substitutions in Triticum aestivum and T. turgidum cultivars
}

\author{
A. Mujeeb-Kazi ${ }^{1}$, M. D. H. M. William and M. N. Islam-Faridi \\ International Maize and Wheat Improvement Center (CIMMYT), Lisboa 27, \\ Apartado Postal 6-641, 06600 México, D. F., Mexico
}

Accepted March 6, 1996

The 1BL/1RS homozygous chromosome translocation involves the short arm of Secale cereale L. chromosome $1 \mathrm{R}(1 \mathrm{RS})$ and the long arm of chromosome $1 \mathrm{~B}$ (1BL) of Triticum aestivum L. The $1 \mathrm{RS}$ chromosome arm possesses leaf, stem, stripe rust and mildew resistance genes (McIntosh 1983, Zeller and Fuchs 1983, Heun and Fischbeck 1987). Presence of the rye arm has been further associated with high yield, stability and wide adaptability of wheat germplasm, specifically for the cultivar Veery cross (Rajaram et al. 1983, Villareal et al. 1994). The Veery lines were derived from the $1 \mathrm{BL} / 1 \mathrm{RS}$ winter bread wheat variety Kavkaz. The translocation was simultaneously and independently identified in Veery "S" by Merker (1982) and Mujeeb-Kazi (1982). Global wheat varietal releases possessing the 1BL/1RS translocation currently occupy over five million hectares of cultivated area. Approximately $40.0 \%$ of our germplasm (Rajaram et al. 1990) and up to $85.0 \%$ of Pakistan wheat varieties in yield trials (Jahan et al. 1990, Ter-Kuile et al. 1991) possess the homozygous 1BL/1RS translocation.

Recently it was suggested (Villareal et al. 1994) that 1BL/1RS wheat varieties exhibit a yield advantage over 1B wheat varieties. Further (Villareal et al. 1995) in a two year yield trial study based upon random $F_{2}$ derived $F_{6}$ advanced lines from a Nacozari (1B)/Seri 82 (1BL/ $1 \mathrm{RS})$ cross concluded that there was a $4.3 \%$ significant yield advantage for the $1 \mathrm{BL} / 1 \mathrm{RS}$ derivatives.

We felt that a more stringent test was necessary and through this report describe the production of germplasm that shall more precisely elucidate the effects of the $1 \mathrm{BL} / 1 \mathrm{RS}$ translocation in a bread and durum wheat variety. This was made possible by substituting in T. aestivum L. cv. Seri 82 (1BL/1RS, 1BL/1RS) a 1B chromosome, and a 1BL/1RS chromosome in $T$. turgidum L. cv. Altar 84 (1B, 1B) by using the backcross procedure. For critical testing of the 1BL effect on yield components the "extracted" Seri 82 and Altar 84 derivatives after eight backcrosses and a selfing were also selected. These "extracted" derivatives may differ from the original breeders Seri 82 and Altar 84 varieties by recombinational changes on the 1BL chromosome arm, a minor but significant feature not met by random $F_{2}$ derived $F_{6}$ germplasm also. The diagnostic biochemical, molecular and cytological procedures used in development of the substituted germplasm are also elucidated.

\section{Materials and methods}

\section{Production and cytological diagnostics}

Triticum aestivum L. cv. Seri $82(1 \mathrm{BL} / 1 \mathrm{RS}, 1 \mathrm{BL} / 1 \mathrm{RS})$ as the female parent was pollinated by T. aestivum L. cv. Pavon 76 (1B, 1B). The resulting Seri $82 \times$ Pavon $_{76} \mathrm{~F}_{1}$ (1BL/1RS, 1B) was backcrossed to Seri 82 . BC $_{1}$ plants were screened by C- or N-banding techniques (Jahan et al. 1990, Jewell and Mujeeb-Kazi 1982) for identifying up to three 1BL/1RS, 1B heterozygote derivative plants which were then each backcrossed to Seri 82 again and similarly

\footnotetext{
${ }^{1}$ Corresponding author: A. Mujeeb-Kazi.
} 
advanced up to backcross VIII $\left(\mathrm{BC}_{8}\right)$; Fig. 1. The $1 \mathrm{BL} / 1 \mathrm{RS}$ transfer into T. turgidum L. cv. Altar 84 (1B, 1B; Fig. 2a) involved a procedure similar to that described above for Seri 82 . Altar 84 , as the female parent, was initially crossed by Seri 82 to generate the $F_{1}$ pentaploid $(2 \mathrm{n}=5 \mathrm{x}=35$, AABBD; $1 \mathrm{BL} / 1 \mathrm{RS}, 1 \mathrm{~B})$. Backcrossing this $\mathrm{F}_{1}$ pentaploid onto Altar 84 gave $\mathrm{BC}$ derivatives from which $2 \mathrm{n}=4 \mathrm{x}=28,1 \mathrm{BL} / 1 \mathrm{RS}, 1 \mathrm{~B}$ plants were identified (Fig. $2 \mathrm{~b}$ ) by chromosome banding and advanced to $\mathrm{BC}_{8}$ (Fig. 3). Selfing after the last backcross $\left(\mathrm{BC}_{8}\right)$ in both cases (Seri 82 and Altar 84 ) gave progeny from which the following categories were selected:

a) Seri 82 with $1 \mathrm{~B}$ homozygous,

b) Seri 82 with $1 \mathrm{BL} / 1 \mathrm{RS}$ homozygous; categorized as 'extracted',

c) Altar 84 with $1 \mathrm{BL} / 1 \mathrm{RS}$ homozygous, and

d) Altar 84 with 1B homozygous; categorized as 'extracted'

\section{Biochemical diagnostics}

Endosperm halves of the germplasm used for fluorescent in situ hybridization (FISH) were utilized for biochemical based identification of $1 \mathrm{BL} / 1 \mathrm{RS}$ homozygotes, 1BL/1RS $+1 \mathrm{~B}$ heterozy-

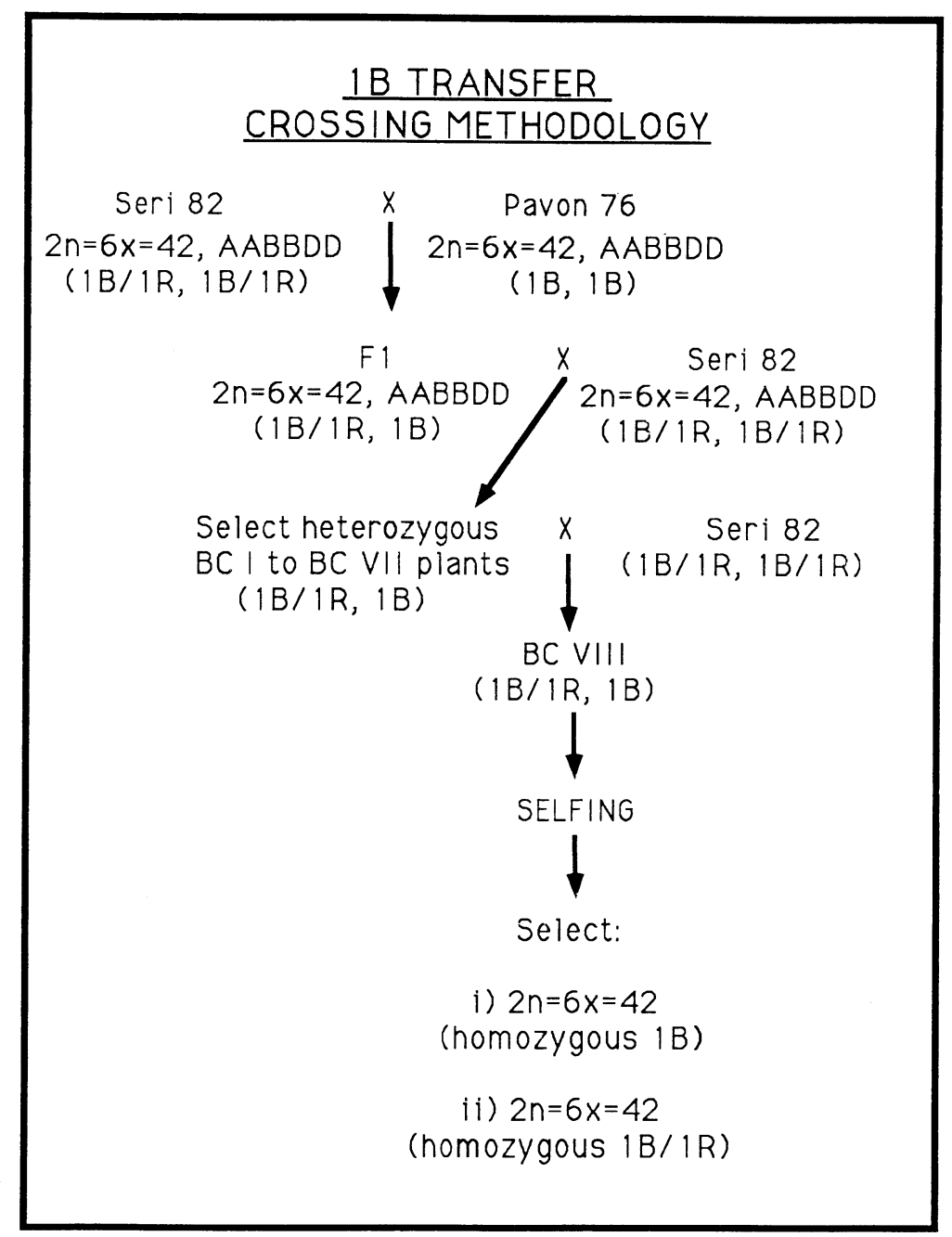

Fig. 1. Crossing methodology for the substitution of chromosome $1 \mathrm{~B}$ for $1 \mathrm{BL} / 1 \mathrm{RS}$ in bread wheat Seri 82 from the cultivar Pavon 76. 


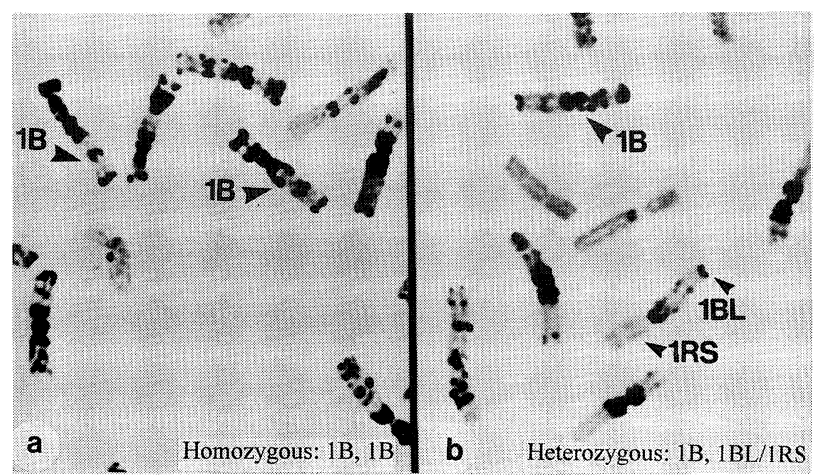

Fig. 2. N-banded partial mitotic spreads of Triticum turgidum cv. Altar 84 showing in (a) homozygosity of chromosome 1B and in (b) heterozygous 1B, 1BL/1RS cell of a plant used in further backcrosses for the substitution of 1BL/1RS in Altar 84 .

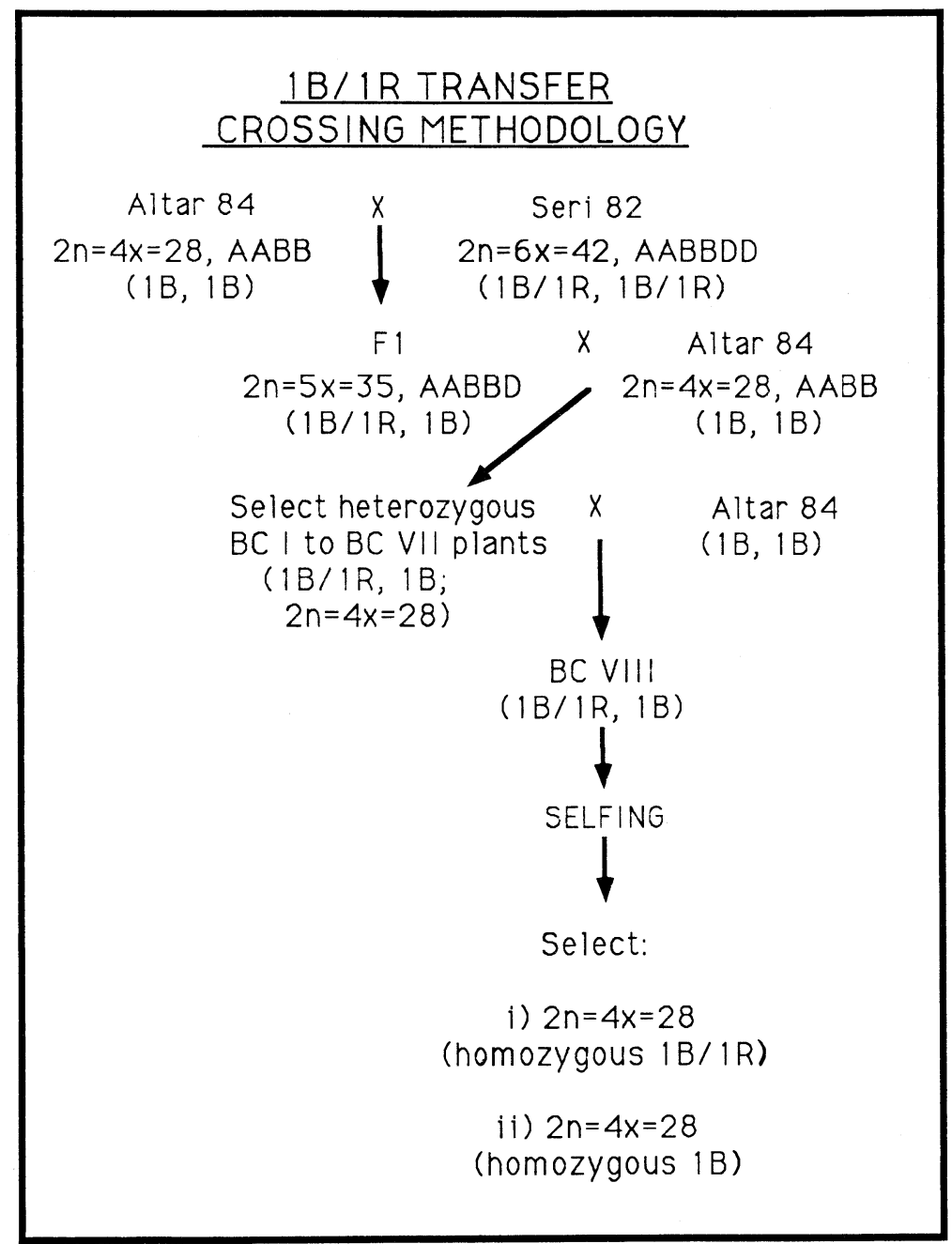

Fig. 3. Crossing methodology for the substitution of chromosome $1 \mathrm{BL} / 1 \mathrm{RS}$ for chromosome $1 \mathrm{~B}$ from a bread wheat Seri 82 to a durum wheat Altar 84 . 
gotes and 1B homozygotes in both Seri 82 and Altar 84 backcross or selfed derivatives. Glucose phosphate isomerase (GPI; Chojecki and Gale 1982) and gliadin polyacrylamide gel electrophoresis (A-PAGE; Bushuk and Zillman 1978) facilitated additional validation of the homozygous $1 \mathrm{BL} / 1 \mathrm{RS}$ and $1 \mathrm{~B}$ as well as the $1 \mathrm{BL} / 1 \mathrm{RS}, 1 \mathrm{~B}$ heterozygote derivatives.

\section{Molecular cytogenetic diagnostics}

The plant material used was Seri 82 (1BL/1RS, 1BL/1RS), Seri $82\left(\mathrm{BC}_{8} 1 \mathrm{BL} / 1 \mathrm{RS}, 1 \mathrm{~B}\right)$, Altar $84\left(\mathrm{BC}_{8} 1 \mathrm{BL} / 1 \mathrm{RS}, 1 \mathrm{~B}\right)$ and Altar $84(1 \mathrm{BL} / 1 \mathrm{RS}, 1 \mathrm{BL} / 1 \mathrm{RS})$. The heterozygotes and homozygotes were ascertained on respective endosperm halves by biochemical assays except for the $1 \mathrm{BL} / 1 \mathrm{RS}, 1 \mathrm{BL} / 1 \mathrm{RS}$ Seri 82 where the commercial variety was utilized. Seed germination, root-tip collection and slide preparation procedures were essentially similar to those of Mujeeb-Kazi and Miranda (1985).

The FISH procedure utilized the published protocols of Schwarzacher et al (1992), Islam-Faridi and Mujeeb-Kazi (1995). S. cereale DNA was biotin labelled by nick translation following instructions accompanying the GIBCO-BRL bionick translation kit. Genomic $T$. aestivum cv. Seri 82 and T. turgidum cv. Altar 84 DNA sheared by autoclaving at 10 psi for 5 min was used 15 times in excess of biotin-14-dATP labelled $S$. cereale genomic DNA. Two stringency washes were done at $37^{\circ} \mathrm{C}$ with $30 \%$ formamide in $0.5 \times \mathrm{SSC}$, followed twice again with $4 \times$ SSC plus Tween-20 $(0.2 \%)$ at room temperature. Biotin labelled $S$. cereale DNA detection utilized fluorescein-avidin coupled with biotinylated anti-avidin for signal amplification using Vector Labs. Inc. protocols.

\section{Results and discussion}

\section{Production and cytological diagnostics}

When C-banded, the $1 \mathrm{BL} / 1 \mathrm{RS}$ chromosome is characterized by prominent bands in the terminal and sub-terminal regions of the short arm (1RS) as well as the centromeric region and terminal region of the long arm (1BL). In contrast, the short arm of chromosome $1 \mathrm{~B}$ of $T$. aestivum shows a striking banding site variation (Figs. $4 \mathrm{a}, \mathrm{b}$ ). Occasionally a faint interstitial band is prevalent in the $1 \mathrm{BL}$ arm of the $1 \mathrm{BL} / 1 \mathrm{RS}$ translocated chromosome. When N-banded, the $1 \mathrm{BL} / 1 \mathrm{RS}$ translocated chromosome can be distinguished from $1 \mathrm{~B}$ because of its unbanded

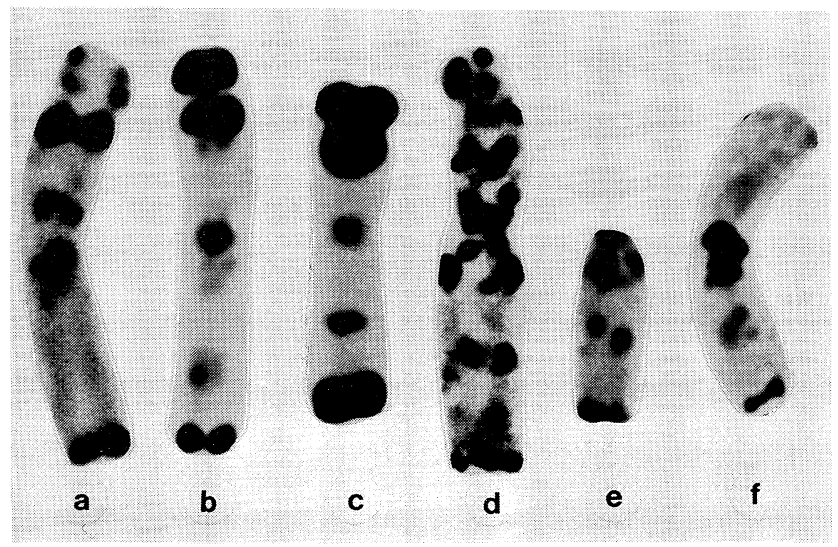

Fig. 4. Chromosome Giemsa banding patterns elucidating the 1RS rye segment from left to right a to $\mathrm{f}$ as follows: a) C-banded $1 \mathrm{~B}$ wheat chromosome, b) C-banded 1BL/1RS translocation chromosome of wheat, c) C-banded $1 \mathrm{R}$ chromosome of Secale cereale, d) N-banded $1 \mathrm{~B}$ wheat chromosome, e) $\mathrm{N}$-banded $1 \mathrm{BL}$ wheat telocentric f) N-banded 1BL/1RS translocation chromosome of wheat. 
1RS arm and copiously banded 1BL characteristics (Figs. 4d, e).

A C-banded 1BL/1RS chromosome can be confused with the $1 \mathrm{R}$ rye chromosome substitution if the cross pedigree of the wheat derivatives is unknown (Figs. 4b, c) and $\mathrm{N}$-banding done to ascertain the rye status. In our case, since the cultivar pedigree of (Seri 82) was identified to possess the $1 \mathrm{BL} / 1 \mathrm{RS}$ translocated chromosome, C-banding alone was used for screening. The Seri $82 \times$ Pavon $76 \mathrm{~F}_{1}$ derivatives of $T$. aestivum were advanced by backcrossing to Seri 82 for producing $\mathrm{BC}_{1}$ progeny. The heterozygote $\mathrm{BC}_{1}$ plants $(1 \mathrm{BL} / 1 \mathrm{RS}, 1 \mathrm{~B})$ were further backcrossed by Seri 82 . Heterozygotes were identified, backcrossed and advanced similarly to $\mathrm{BC}_{8}$. This $\mathrm{BC}_{8}$ progeny was selfed and plants homozygous for $1 \mathrm{~B}$ and $1 \mathrm{BL} / 1 \mathrm{RS}$ selected. Both categories of plants in general resembled Seri 82 in phenotype, grain color, maturity and height.

\section{Biochemical diagnostics}

The GPI genes are located on the short arms of the homoeologous group I chromosomes of wheat (Hart 1979), and the gene product of chromosome 1BS is observed as the most cathodal band on GPI zymograms. This band is marked for the homozygous 1B wheat cultivar Pavon (Fig. 5a) and in the heterozygous 1B, 1BL/1RS Seri 82/Pavon $F_{1}$ hybrid (Fig. 5b). The band is missing in the homozygous $1 \mathrm{BL} / 1 \mathrm{RS}$ cultivar Seri 82 (Fig. 5c). When the 1B, 1BL/1RS heterozygotes are backcrossed by the recurrent 1BL/1RS homozygous parent Seri 82, progeny is produced that is $1 \mathrm{BL} / 1 \mathrm{RS}, 1 \mathrm{BL} / 1 \mathrm{RS}$ or $1 \mathrm{~B}, 1 \mathrm{BL} / 1 \mathrm{RS}$. GPI screening of individual endosperm halves detects the $1 \mathrm{BL} / 1 \mathrm{RS}$ homozygous progeny which is eliminated whereas the 1B, 1BL/1RS heterozygotes are retained for further backcrosses with Seri 82 until eight are completed (Fig. 1 schematic).

After eight backcrosses a selfing of the heterozygote plants is required for separating the homozygous 1B substitution, homozygous 1BL/1RS extracted Seri 82, and the critical 1B, 1BL/ $1 \mathrm{RS}$ progeny that is identical to the homozygous 1B Seri 82 banding pattern. These heterozygotes have to be identified and discarded. Endosperm halves are subjected to GPI analyses that separates the $1 \mathrm{BL} / 1 \mathrm{RS}$ extracted Seri 82 progeny. The remaining endosperm halves which could represent $1 \mathrm{~B}$ homozygotes or the $1 \mathrm{~B}, 1 \mathrm{BL} / 1 \mathrm{RS}$ are subjected to A-PAGE analyses. Seri 82 progeny homozygous for the $1 \mathrm{~B}$ substitution is separated since it lacks the rye secalin bands

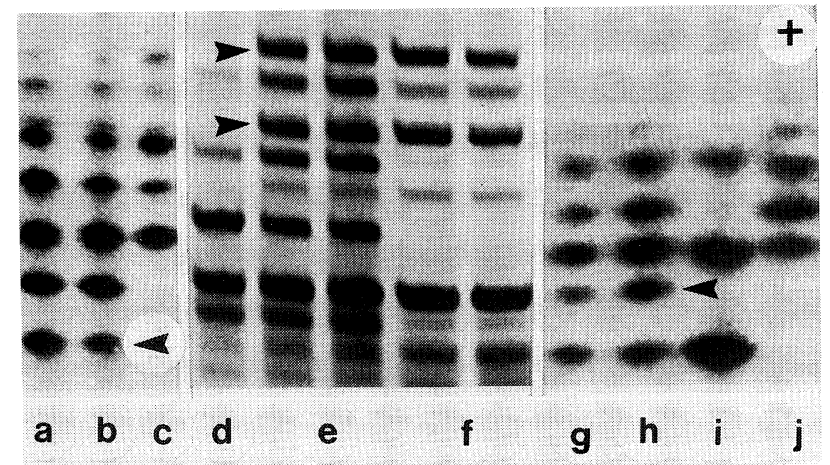

Fig. 5. - Glucose phosphate isomerase banding pattern of grain from (a) Triticum aestivum cv. Pavon "S" (1B, 1B), (b) F1 of cv. Pavon/Seri 82 (1B, 1BL/1RS), (c) Cultivar Seri 82 (1BL/ $1 \mathrm{RS}, 1 \mathrm{BL} / 1 \mathrm{RS})$. Marker band for the $1 \mathrm{~B}$ short arm indicated by an arrow. - Gliadin A-PAGE patterns of the cultivar Seri 82 (1B, 1B) (d), two tracks of the Seri (1B, 1BL/1RS) F1 (e) and Seri 82 (1BL/1RS, 1BL/1RS) cultivar (f). The rye (1RS) marker bands are arrowed and are within the omega-gliadin region. - GPI banding patterns on IEF polyacrylamide gels for $T$. aestivum cv. Chinese Spring (1B, 1B) (g), a durum based 1B, 1BL/1RS F1 (h) T. turgidum cv. Altar 84 (1B, 1B) (i) and a durum 1BL/1RS, 1BR/1RS translocation line (j). The arrow identified the 1B, 1BL/1RS heterozygote band. 
(Fig. 5d). By GPI Seri 82 homozygous for 1BL/1RS derivatives had already been separated and A-PAGE unequivocally identifies the homozygous $1 \mathrm{~B}$ derivatives. A-PAGE tracks that show the secalin bands (Fig. 5e) are of heterozygotes and discarded.

Wheat cultivars homozygous for chromosome $1 \mathrm{~B}$ have a GPI zymogram pattern (Fig. $5 \mathrm{~g}$ ) that is identical to that of a $1 \mathrm{~B}, 1 \mathrm{BL} / 1 \mathrm{RS}$ heterozygote durum wheat (Fig. $5 \mathrm{~h}$ ). The arrowed diagnostic band in the heterozygote is contributed by the 1RS arm. This is consistent with the findings of Chojecki and Gale (1982) that suggested the GPI-R $_{1}$ subunits behaved similar to the GPI- $D_{1}$ units. The $1 B, 1 B L / 1 R S 2 n=4 x=28$ heterozygotes were advanced by eight backcrosses to the durum 1B cultivar Altar 84 recurrent parent. Over this advance GPI analyses enabled identification of the $1 \mathrm{~B}$ homozygotes that were discarded. After reaching the $\mathrm{BC}_{8}$ generation the heterozygotes were selfed. The GPI analyses on individual endosperm halves identified 1B homozygous altar 84 derivatives categorized as "extracted" Altar 84 . The corresponding embryo halves of these seeds were seed increased. The remaining embryo halves were germinated and root-tips analyzed by Giemsa C-banding to identify plants that were $1 \mathrm{BL} /$ 1RS homozygotes. The heterozygote seedlings were discarded.

\section{Molecular cytogenetic diagnostics}

We utilized the FISH molecular diagnostics to ascertain that the $\mathrm{BC}_{8}$ selfed derivatives
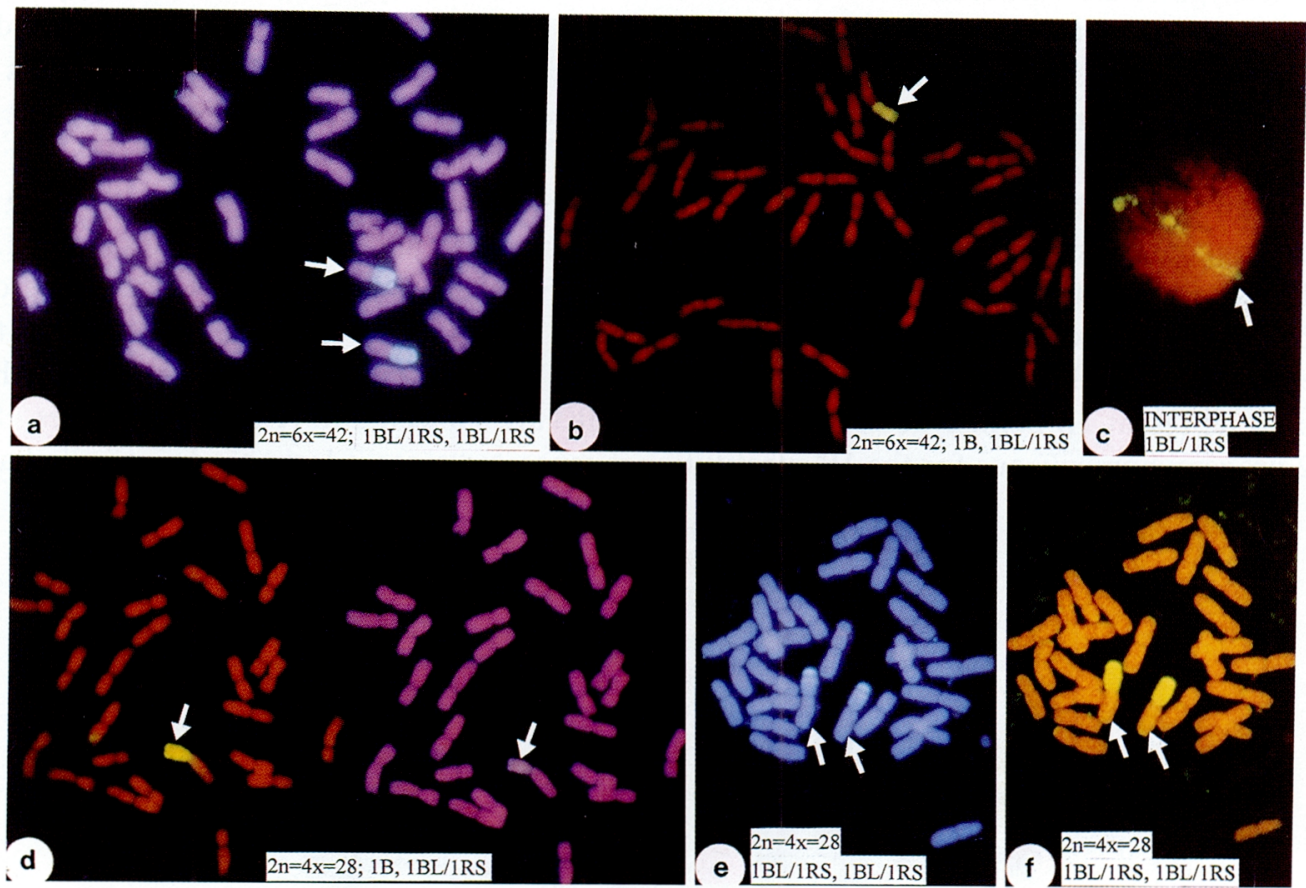

Fig. 6. Fluorescent in situ hybridization micrographs using filter sets 02,09 and 23 of a Zeiss epifluorescence research microscope associated with identification of the 1BL/1RS translocation chromosome in bread and durum wheat germplasm. Photographs on a Fujicolor super HG400 print film. (a) $T$. aestivum cv. Seri 82 homozygous 1BL/1RS (DAPI stain, filter 02) chromosomes arrowed, (b) A backcross heterozygote where Seri 82 is the recurrent parent. The 1BL/ 1RS heterozygote arrowed (filter 23), (c) The 1BL/1RS heterozygote of a BC Seri 82 in an interphase mitotic cell (Filter 23), (d) a backcross heterozygote where the durum wheat Altar 84 is the recurrent parent. Cell photographs under filter 23 (left) and 02 (right) show the single $1 \mathrm{BL} / 1 \mathrm{RS}$ chromosome in the $2 \mathrm{n}=4 \mathrm{x}=28$ complement, (e and $\mathrm{f}$ ) Selfing of the $\mathrm{BC}_{8}$ Altar heterozygote (1B, 1BL/1RS) showing the homozygous $1 \mathrm{BL} / 1 \mathrm{RS}$ derivative. Mitotic cell photographed under filters 02 and 09 respectively with the two 1BL/1RS chromosomes arrowed. 
complemented the inferences obtained from the cytological and biochemical data about chromosomal uniformity of physical structure. There appears to be no visible structural change in the $1 \mathrm{RS}$ segment of the $1 \mathrm{BL} / 1 \mathrm{RS}$ translocation (Figs. $6 \mathrm{a}-\mathrm{f}$ ). The homozygous $1 \mathrm{BL} / 1 \mathrm{RS}$ parents Seri 82 and the substituted derivatives of Altar 84 characterize the centric-break association that produced the $1 \mathrm{BL} / 1 \mathrm{RS}$ translocation (Figs. 6a, e, f). The 1B, 1BL/1RS heterozygotes (Figs. 6b, d) also represent the normal 1RS arm location, and this single rye chromosome arm is elaborately illustrated in mitotic interphase (Fig. 6c).

\section{Summary}

Wheat (Triticum aestivum L.) varieties with the 1BL/1RS chromosome translocation exhibit a major role in agricultural production, partly attributed to their high yield advantage. The evidence as to whether the 1RS segment may have genetically contributed to this yield advantage has not been unequivocally demonstrated due to lack of appropriate testor germplasm. This analysis now appears possible through the testing of a bread wheat (T. aestivum $\mathrm{L}$. cv. Seri 82) and a durum wheat variety (T. turgidum L. cv. Altar 84) in which their homozygous $1 \mathrm{BL} / 1 \mathrm{RS}$ and $1 \mathrm{~B}$ chromosomes have been substituted by $1 \mathrm{~B}$ and $1 \mathrm{BL} / 1 \mathrm{RS}$ chromosomes respectively. Heterozygote (1BL/1RS, 1B) $F_{1}$ and backcross derivatives were advanced by eight backcrosses respectively to Seri 82 or Altar 84 with an ultimate selfing to obtain homozygous 1B Seri 82 and 1BL/1RS Altar 84 derivatives. Diagnostic procedure for tracking the $1 \mathrm{BL} / 1 \mathrm{RS}$ chromosomes in heterozygous and homozygote derivatives utilized Giemsa C- or N-banding differential staining, electrophoretic biochemical assays and FISH.

\section{References}

Bushuk, W. and Zillman, R. R. 1978. Wheat cultivar identification by gliadin electrophoregrams. I. Apparatus, method and nomenclature. Can. J. Plant. Sci. 58: 505-515.

Chojecki, A. J. S. and Gale, M. D. 1982. Genetic control of gluclose phosphate isomerase in wheat and related species. Heredity 49: 337-347.

Hart, G. E. 1979. Evidence for a triplicate set of glucosephosphate isomerase structural genes in hexaploid wheat. Biochem. Genet. 17: 585-598.

Heun, M. and Fishbeck, G. 1987. Identification of wheat powdery mildew resistance genes by analyzing host-pathogen interactions. Plant Breed. 98: 124-129.

Islam-Faridi, M. N. and Mujeeb-Kazi, A. 1995. Visualization by fluorescent in situ hybridization of Secale cereale DNA in wheat germplasm. Theor. Appl. Genet. 90: 595-600.

Jahan, Q., Ter-Kuile, N., Hashmi, N., Islam, M., Vahidy, A. A. and Mujeeb-Kazi, A. 1990. The status of the 1B/1R translocation chromosome in some released wheat varieties and the 1989 candidate varieties of Pakistan. Pak. J. Bot. 22: 1-10.

Jewell, D. and Mujeeb-Kazi, A. 1982. Unexpected chromosome numbers in backcross-I generations of $F_{1}$ hybrids between Triticum aestivum and related alien genera. Wheat Inf. Serv. 55: 5-9.

McIntosh, R. W. 1983. A catalogue of gene symbols. In: Proc 6th Int Wheat Genet Symp, Kyoto, Japan. S. Sakamoto (ed), Kyoto University, pp. 1197-1255.

Merker, A. 1982. "Veery" - a CIMMYT spring wheat with 1B/1R chromosome translocation. Cer. Res. Comm. 10: 105-106.

Mujeeb-Kazi, A. 1982. CIMMYT report on wheat improvement, 78-87.

- and Miranda, J. L. 1985. Enhanced resolution of somatic chromosome constriction as an aid to identifying intergeneric hybrids among some Triticeae. Cytologia 50: 701-709.

Rajaram, S., Mann, Ch. E., Ortiz-Ferrara, G. and Mujeeb-Kazi, A. 1983. Adaptation, stability and high yield potential of certain 1B/1R CIMMYT wheats. In: Proc 6th Int Wheat Genet Symp, Kyoto, Japan. S. Sakamoto (ed), Kyoto University, pp. 613-621.

—, Villareal, R. L. and Mujeeb-Kazi, A. 1990. The global impact of 1B/1R spring wheats. Agr. Abst. Amer. Soc. Agr., Madison, Wisconsin, USA. p. 106.

Schwarzacher, T., Anamthawat-Jónsson, K., Harrison, G. E., Islam, A. K. M. R., Jia, J. S., King, I. P., Leitch, A. R., 
Miller, T. E., Reader, S. M., Rogers, W. J., Shi, M. and Heslop-Harrison, J. S. 1992. Genomic in situ hybridization to identify alien chromosomes and chromosome segments in wheat. Theor. Appl. Genet. 84: 778-786.

Ter-Kuile, N., Jahan, Q., Hashmi, N., Aslam, M., Vahidy, A. A. and Mujeeb-Kazi, A. 1991. 1B/1R translocation wheat cultivars detected by A-PAGE electrophoresis and C-banding in the 1990 National Uniform Wheat Yield Trial in Pakistan. Pak. J. Bot. 23: 203-212.

Villareal, R. L., Mujeeb-Kazi, A., Rajaram, S. and Del-Toro, E. 1994. Associated effect of chromosome 1B/1R translocation on agronomic traits in hexaploid wheat. Breed. Sci. 44: 7-11.

-, Del-Toro, E., Mujeeb-Kazi, A. and Rajaram, S. 1995. The 1BL/1RS chromosome translocation effect on yield characteristics in a Triticum aestivum L. cross. Pl. Breeding 114: 497-500.

Zeller, F. J. and Fuchs, E. 1983. Cytology and disease resistance of a 1A/1R wheat-rye translocation cultivar. Z. Pflanzenzucht. 90: 285-296. 